\title{
Effect of inner physical properties on powder adhesion in inhalation capsules in case of a high resistance device
}

\author{
HIROKO OTAKE* , MISA MINAMI* , MIZUKI YAMAGUCHI, SAWAKO AKIYAMA, \\ KAZUNORI INABA and NORIAKI NAGAI
}

Faculty of Pharmacy, Kindai University, Higashi-Osaka, Osaka 577-8502, Japan

Received May 15, 2021; Accepted August 20, 2021

DOI: $10.3892 /$ etm.2021.10788

\begin{abstract}
The inhalation performance of a dry powder inhaler (DPI) depends on the inhalation patterns of patients, inhalation particle characteristics and inhalation devices. In capsule-based DPIs, the capsule plays an important role in the dispersion of inhalation particles. The present study investigated the effects of inner physical properties of capsules on drug release from capsules-based DPIs with high resistance device. Atomic force microscopy (AFM) was used to evaluate the capsule physical properties, such as the capsule inner structure and surface potential, of three capsules with different compositions (G-Cap, PEG/G-Cap, and HPMC-Cap). As a model dry powder for capsule-based DPIs, the dry powder in Spiriva ${ }^{\circledR}$ Inhalation Capsules containing tiotropium bromide was used. Inhalation performance was evaluated using a twin-stage liquid impinge and Handihaler ${ }^{\circledR}$ (flow rate $30 \mathrm{l} / \mathrm{min}$ ). The results indicated that the capsule inner surface presented with numerous valleys and mountains, regardless of the capsule type. Furthermore, the valley and mountain areas
\end{abstract}

Correspondence to: Dr Noriaki Nagai, Faculty of Pharmacy, Kindai University, 3-4-1 Kowakae, Higashi-Osaka, Osaka 577-8502, Japan

E-mail: nagai_n@phar.kindai.ac.jp

*Contributed equally

Abbreviations: AFM, atomic force microscopy; COPD, chronic obstructive pulmonary disease; DPI, dry powder inhaler; ED, emitted dose; FPD, fine particle dose; FPF, fine particle fraction; GSD, geometric standard deviation; HPLC, high performance liquid chromatography; HPMC, hydroxypropyl methylcellulose; KFM, kelvin probe force microscopy; LOD, loss of drying; MB, mass balance; MMAD, mass median aerodynamic diameter; RD, recovery dose; $\mathrm{RH}$, relative humidity; SEM, scanning electron microscopy; SPM, scanning probe microscopy; TIO, dry powder in Spiriva $^{\circledR}$ inhalation capsules containing tiotropium bromide; TSLI, twin-stage liquid impinger

Key words: capsule-based dry powder inhaler, capsule inner surface, physical property, capsule composition, surface potential, atomic force microscopy, high resistance device on the capsule inner surface showed a significantly higher or lower surface potential. Following inhalation of capsule-based DPIs, the drug remained in the valleys on the capsule inner surface; however, no significant difference was observed in the drug release from capsule and lung drug delivery. Therefore, inhalation performance in capsule-based DPIs when a high resistance device, such as Handihaler ${ }^{\circledR}$, is used at an appropriately flow rate is not markedly affected by the physical properties of the capsule inner surface due to capsule composition.

\section{Introduction}

Inhalation drug products have been developed for the treatment of respiratory diseases, such as asthma, chronic obstructive pulmonary disease (COPD) and pulmonary infections. Inhalation drug products are also used to treat systemic diseases, including diabetes $(1,2)$, and their application for pulmonary vaccination (3) is expected. Three types of inhalation drug products are currently on the market: nebulizers, pressurized metered dose inhalers (pMDIs) and dry powder inhalers (DPIs). In contrast to nebulizers and pMDIs, DPIs deliver a powder formulation without synchronizing the timing of nebulization and inhalation. Furthermore, a nebulizer and propellant are not necessary for DPI and the device is small. However, drug release from DPIs is affected by the inhalation patterns of patients (4-7), inhalation devices (5), inhalation formulations (8) and the physiology of the airways (9). There are two types of DPIs, the multi- and single-unit-dose inhalers (10-12). A typical example of a single-unit-dose inhaler is capsule-based DPIs, including Intal ${ }^{\circledR}$ Spinhaler $^{\circledR}$, Spiriva ${ }^{\circledR}$ Handihaler $^{\circledR}$, Onbrez ${ }^{\circledR}$, Ultibro ${ }^{\circledR}$ and Seebri ${ }^{\circledR}$ Breezhaler $^{\circledR}$. These inhalers are widely distributed as commercial inhalers. The advantages of capsule-based DPIs are the accurate and uniform drug delivery as well as the simplicity of use for patients (13). Patients can also visually confirm whether a dose has been administered (14). Drug retention in capsules affects inhalation performances and attenuates therapeutic efficacy for pulmonary diseases $(15,16)$. Therefore, the development of a formulation with controlled drug release from capsules is needed for capsule-based DPIs. To improve drug release from capsules, previous studies focused on inhalation particles. For example, drug release from capsules is improved by preparing drug-carrier particles and drug-drug granulate particles or adding dispersive 
agents, including L-leucine, L-phenylalanine, DPPC and DOTAP. These inhalation particles preparation techniques attenuate drug-drug and drug-capsule adhesive-cohesive forces (17-21). The capsule body has also been previously investigated. Wauthoz et al (22) and Saleem et al (23) have reported that inhalation characteristics depend on capsule compositions [e.g., gelatin and hydroxypropyl methylcellulose (HPMC)], capsule loss on drying, capsule piercing pattern, the manufacturing method and the lubricant content. Most of these findings were related to the capsule outer properties; however, there are few studies on the effect of capsule inner properties. In addition, most of the previous studies have been performed using a low resistance device instead of a high resistance device.

The present study aimed to evaluate the effects of capsule inner surface physical properties on drug release from capsules and lung drug delivery using a high resistance device.

\section{Materials and methods}

Materials. As a model capsule-based DPIs, Spiriva ${ }^{\circledR}$ Handihaler ${ }^{\circledR}$ was purchased from Boehringer Ingelheim Japan Ltd. Spiriva ${ }^{\circledR}$ Handihaler ${ }^{\circledR}$ is a typical capsule-based DPIs, used for the treatment of COPD and for reducing COPD exacerbations. A dry powder in Spiriva ${ }^{\circledR}$ inhalation capsules containing tiotropium bromide (TIO) as the main component was used as a model drug. The capsule contained $5.5 \mathrm{mg}$ of a powder formulation consisting of $18 \mu \mathrm{g}$ micronized tiotropium (as bromide hydrate, $22.5 \mu \mathrm{g}$ ) with coarse lactose monohydrate (24). The Handihaler ${ }^{\circledR}$ (Boehringer Ingelheim Japan Ltd.) was used as a model high resistance device for capsule-based DPIs (device specific resistance $\left.3.03 \mathrm{~Pa}^{*} \mathrm{~min}^{2} / 1^{2}\right)(25)$. Size 3 capsules with different compositions were supplied by Qualicaps, Co., Ltd. (G-Cap, gelatin capsule; PEG/G-Cap, gelatin capsule containing 5\% PEG4000 as plasticizer; and HPMC-Cap, HPMC capsule). $\mathrm{PEG} / \mathrm{G}-\mathrm{Cap}$ is a gelatin capsule that is resistant to cracking due to the presence of PEG. HPMC-Cap is resistant to cracking under dry conditions and may be filled with highly hygroscopic drugs. Each capsule was stored at $15-25^{\circ} \mathrm{C}$ and under $40-50 \%$ relative humidity $(\mathrm{RH})$ in a glass desiccator. The characteristics of each capsule are presented in Table I. All other reagents and solvents were of analytical or HPLC grade.

Characterization of three type of capsule with different compositions. Each capsule image was analyzed using the image analysis software ImageJ, and the length diameter, width diameter, surface area and volume were calculated (Table I). The loss on drying (LOD) and lubricant content of each capsule are shown as stated in the analysis certificate. The numbers shown in Table I correspond to the average of three capsules of each capsule type except for LOD and lubricant content. Fig. 1A-C display the appearance of each capsule.

Morphological analysis of TIO by scanning electron microscopy. The morphology of TIO was examined using scanning electron microscopy (SEM; SU1510; Hitachi High-Technologies Corp.). Prior to observations, free falling powders from a spatula were manually dispersed on a specimen mount with double-side tape and were then coated with platinum using an ion sputter coater (E-1013; Hitachi High-Technologies Corp.).

Capsule hole examination after piercing in Handihaler ${ }^{\circledR}$. Ten capsules of each type were successively placed in the Handihaler ${ }^{\circledR}$ to be pierced. Capsule hole images were taken of the most representative hole type of each capsule. In addition, each capsule hole image was analyzed using the image analysis software ImageJ (version 1.8.0_172; National Institutes of Health), and the hole diameter and hole area were calculated.

Topographical analysis of capsule inner surface by AFM. AFM is widely used for micro- and nanoscale material conditions to create a 3D image of a physical surface (26). In the present study, the AFM topography of each capsule inner surface was obtained by scanning probe microscopy (SPM-9700HT; Shimadzu Corp.). Prior to observations, the capsule was cut off and placed on a specimen mount with double-side tape. AFM topography was performed in air using an EFM cantilever with a force constant $42 \mathrm{~N} / \mathrm{m}$ in the phase mode. All experiments were performed in triplicate with an area of $20.0 \times 20.0 \mu \mathrm{m}$ in each capsule. The obtained AFM images were evaluated and quantified using ImageJ software. The capsule inner surface of each capsule using AFM was characterized by calculating the valley diameter, the valley area and the valley ratio. The valley diameter corresponds to the average diameter of capsule valleys in the total observed area. The valley area is the average area of capsule valleys in the total observed area. The valley ratio was expressed as the ratio of valley areas in the total observed area. The valley diameter, valley area and valley ratio were evaluated for 40 valleys in one observation area, and the average value was calculated.

Measurement of capsule inner surface potential distribution with the KFM mode. The potential distribution of each capsule inner surface was obtained using the KFM mode from SPM-9700HT. The KFM mode is a method used to measure the potential distribution of the sample surface. This distribution is evaluated by detecting the electrostatic force acting between the sample surface and the probe tip by applying an alternating current signal to the electric conductive cantilever. A KFM image is obtained in air using the EFM cantilever with a force constant of $2.8 \mathrm{~N} / \mathrm{m}$ in KFM mode. Prior to observation, the capsule was cut off and placed on a specimen mount with double-side tape. All experiments were performed in triplicate with an area of $5.0 \times 5.0 \mu \mathrm{m}$ in each capsule. Image analysis was performed on a representative image of each capsule. Images were analyzed using SPM-9700HT manager software (Shimadzu Corp.).

In vitro inhalation performance evaluation. Inhalation performance was evaluated using a twin-stage liquid impinger (TSLI; European Pharmacopoeia Apparatus; Copley Scientific Ltd.) equipped with a suction pump. Stages 1 and 2 in TSLI contained 7 and $30 \mathrm{ml}$ of purified water and methanol (75:25, volume ratio), respectively. Each capsule (G-Cap, PEG/G-Cap and HPMC-Cap) was manually filled with $5.5 \pm 0.1 \mathrm{mg}$ of TIO while the weight was measured using sensitive digital balance ( $\pm 0.1 \mathrm{mg}$; Balance XS64; Mettler-Toledo $\mathrm{GmbH})$. Once Handihaler ${ }^{\circledR}$ was connected to the mouthpiece of TSLI, the 
Table I. Characteristics of each capsule.

\begin{tabular}{lccc}
\hline & G-Cap & PEG/G-Cap & HPMC-Cap \\
\cline { 2 - 4 } Main material & Gelatin & Gelatin, PEG4000 & Hydroxypropyl methyl cellulose \\
\hline Length diameter, mm & $12.7 \pm 1.53$ & $13.9 \pm 0.79$ & $14.1 \pm 0.56$ \\
Width diameter, mm & $4.37 \pm 0.53$ & $4.67 \pm 0.14$ & $4.52 \pm 0.27$ \\
Surface area, mm m $^{2}$ & $289 \pm 6.14$ & $245 \pm 20.5$ & $268 \pm 24.6$ \\
Volume, mm mm $^{3}$ & $304 \pm 1.03$ & $301 \pm 2.04$ & $313 \pm 17.9$ \\
Loss of drying, $\%$ & 14.3 & 13.2 & 5.0 \\
Lubricant content & $\mathrm{NR}$ & $\mathrm{NR}$ & NR
\end{tabular}

Each value represents the mean \pm standard error of measurements. $n=3$. 'Lubricant content' of each capsule is displayed as 'NR'. This is because there was no detailed description in the analysis certificate. G-Cap, gelatin capsule; PEG/G-Cap, gelatin capsule containing 5\% PEG4000 as plasticizer; HPMC-Cap, hydroxypropyl methylcellulose capsule; NR, not reported.
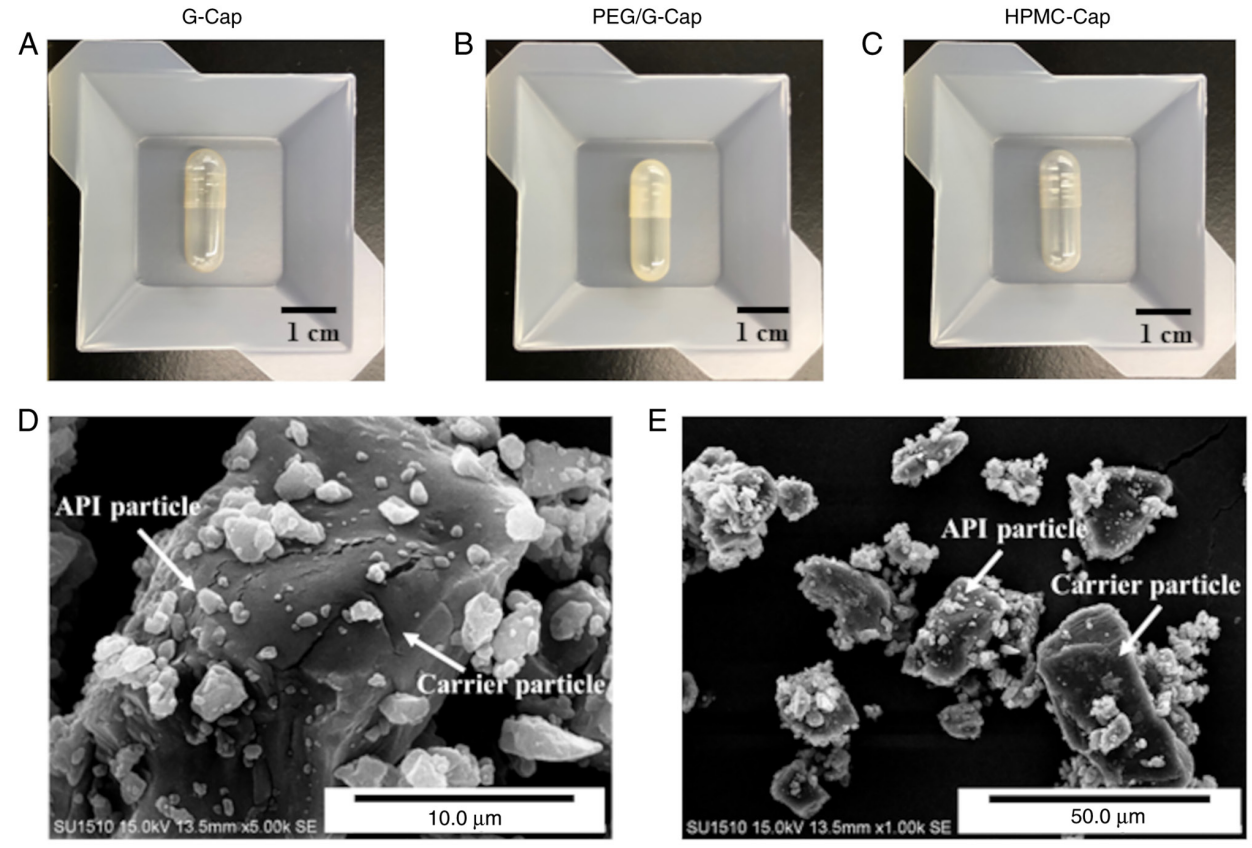

Figure 1. Images of the (A-C) three capsule types and (D and E) SEM images of TIO. (A) G-Cap, (B) PEG/G-Cap, and (C) HPMC-Cap. (D) High magnification, x5000 image of TIO. (E) Low magnification, x1,000 image of TIO. G-Cap, gelatin capsule; PEG/G-Cap, gelatin capsule containing 5\% PEG4000 as plasticizer; HPMC-Cap, hydroxypropyl methylcellulose capsule; API, active pharmaceutical ingredient; TIO, tiotropium bromide.

TIO-loaded capsule was placed in the holder of Handihaler ${ }^{\circledR}$ with a pin to pierce it. The Handihaler ${ }^{\circledR}$ pierced one hole in the side wall of the cap and the body. The suction pump was operated to disperse the powder in the capsule at a flow rate $30 \mathrm{l} / \mathrm{min}$. Since the lower limit of peak inhalation flow rates for Handihaler ${ }^{\circledR}$ is $>201 / \mathrm{min}(25)$, the flow rate of the suction pump was set to $30 \mathrm{l} / \mathrm{min}$ in the present study. Dry powder remaining in the capsule and device at each stage after dispersion by the suction pump was collected by rinsing with purified water and methanol (75:25, volume ratio). The collected samples were diluted into $50 \mathrm{ml}$ purified water and methanol (75:25, volume ratio), and the concentration of TIO in each sample was measured by high performance liquid chromatography (HPLC). All experiments were performed in triplicate. The inhalation performance of TIO by TSLI was characterized using the following parameters: i) Recovered dose (RD) is the mass of TIO recovered from all parts of the apparatus (capsule, device and TSLI); ii) mass balance (MB) is the ratio between RD to the amount of TIO to be loaded into capsule; iii) drug retention for capsule is the ratio of the amount of capsule drug deposition of the RD, while Emitted dose (ED) is the amount of drug particles emitted from a capsule to that from an inhalation device; iv) fine particle dose (FPD) is the mass of drug particles from Stage 2 within the RD; and v) fine particle fraction (FPF) is the ratio between FPD to RD.

Conditions for measuring TIO by HPLC. The concentration of TIO in in vitro inhalation characteristics samples was determined using Shimadzu LC-20AT system (Shimadzu Corp.), which consists of a UV-vis detector (SPD-20A), 

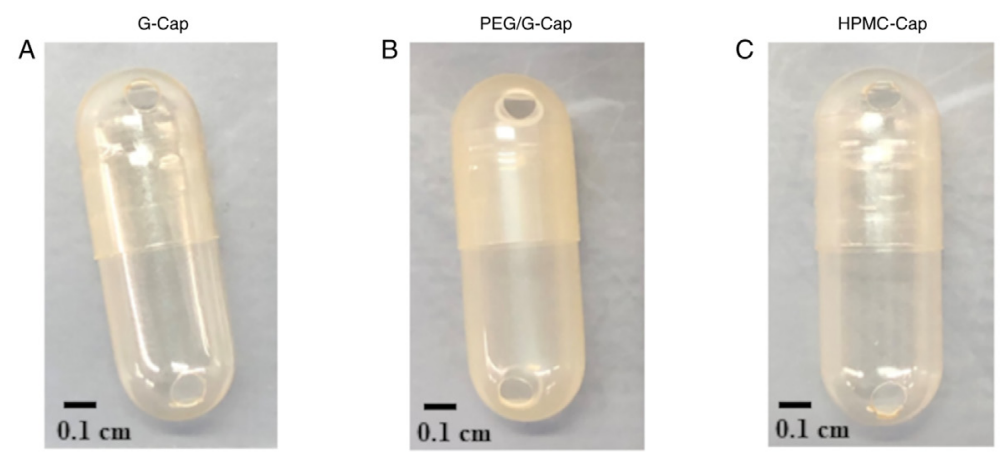

Figure 2. Image of hole type after piercing by Handihaler ${ }^{\circledast}$. (A) G-Cap, (B) PEG/G-Cap, and (C) HPMC-Cap. G-Cap, gelatin capsule; PEG/G-Cap, gelatin capsule containing 5\% PEG4000 as plasticizer; HPMC-Cap, hydroxypropyl methylcellulose capsule.

Table II. Characteristics of pierced capsule holes of each capsule.

Hole diameter, $\mathrm{mm}$

Hole area, $\mathrm{mm}^{2}$

\begin{tabular}{lll}
\hline G-Cap & $1.28 \pm 0.02$ & $1.56 \pm 0.08$ \\
PEG/G-Cap & $1.14 \pm 0.98$ & $1.26 \pm 0.06$ \\
HPMC-Cap & $1.17 \pm 0.15$ & $1.35 \pm 0.19$
\end{tabular}

Each value represents the mean \pm standard error of measurements. $\mathrm{n}=10$. G-Cap, gelatin capsule; PEG/G-Cap, gelatin capsule containing 5\% PEG4000 as plasticizer; HPMC-Cap, hydroxypropyl methylcellulose capsule.

column oven (CTO-20A), degassing unite (DGU-20A $\mathrm{A}_{3 \mathrm{R}}$ ) and an auto sampler (SIL-10AF). The mobile phase was composed of $100 \mathrm{mM}$ potassium dihydrogenphosphate solution ( $\mathrm{pH} 4.0)$ and acetonitrile (80:20, volume ratio). The flow rate was set to $0.25 \mathrm{ml} / \mathrm{min}$. The column (Inertsil ${ }^{\mathbb{E}}$ ODS-3, 3.0 $\mu \mathrm{m}, 2.1 \times 50 \mathrm{~mm}$; GL Science Co., Inc.) was heated at $35^{\circ} \mathrm{C}$. The injection volume was $100 \mu \mathrm{l}$. The UV absorbance of each sample was measured at $238 \mathrm{~nm}$. Under these HPLC condition, the limit of detection for TIO was $0.03 \mu \mathrm{g} / \mathrm{ml}$, and a calibration curve was linear at a concentration range of $0.1-20 \mu \mathrm{g} / \mathrm{ml}$. The correlation coefficients $\left(\mathrm{R}^{2}\right)$ was 0.999 $(y=326.12 x+2684.8)$. All samples were properly diluted to fit within this calibration range and peak area was used for quantification of TIO.

Statistical analysis. All data were presented as the means \pm standard errors of measurements. The 'BellCurve for Excel' (Social Survey Research Information Co., Ltd.) was used for statistical data analysis. One-way ANOVA followed by Dunnett's post hoc test for multiple comparison and an unpaired Student's t-test were used for statistical analyses. $\mathrm{P}<0.05$ was considered to indicate a statistically significant difference.

\section{Results}

Capsule characterization. The characterization of the three type of capsules (G-Cap, PEG/G-Cap and HPMC-Cap) are presented in Table I. The appearance of each capsule is shown in Fig. 1A-C. G-Cap and HPMC-Cap were clear capsule bodies, whereas PEG/G-Cap had a translucent capsule body. No significant differences were observed in the length diameter, width diameter, surface area and volume of each capsule between G-Cap, PEG/G-Cap and HPMC-Cap [non-significant (NS), ANOVA]. The LOD of HPMC-Cap was much lower than the LOD of G-Cap and PEG/G-Cap, with no difference between G-Cap to PEG/G-Cap (Table I).

Morphology of a model dry powder. In the present study, TIO was used as a dry powder model for capsule-based DPIs. TIO consists of micronized tiotropium particles and coarse lactose monohydrate particles as carriers. The morphology of TIO is presented in Fig. 1D and E. The SEM images clearly demonstrated that micronized tiotropium particles [active pharmaceutical ingredient (API) particles] adhered to coarse lactose monohydrate particles (carrier particles).

Pierced capsule hole characterization. The image of capsule hole of each capsule is presented in Fig. 2. The characteristics of pierced capsule holes of each capsule are presented in Table II. In terms of capsule hole after piercing, no cracked holes between G-Cap, PEG/G-Cap and HPMC-Cap were seen (Fig. 2). The capsule hole diameter and hole area of G-Cap tended to be larger than that of PEG/G-Cap and HPMC-Cap (Table II; NS, ANOVA).

Comparison of inner surface structure of each capsule. Fig. 3 presents the inner surface structure of each capsule before TIO loading and after suction of TIO-loaded capsules. The valley diameter, valley area and valley ratio of capsule inner surface before TIO loading and after the suction of TIO-loaded capsules are shown in Table III. In Fig. 3A-C, the areas darker than background indicated the valleys while the lighter areas indicated the mountains. Numerous valleys and mountains were observed on the capsule inner surface regardless of the capsule composition. After the suction of TIO-loaded capsules, TIO was observed in valleys on the capsule inner surface (Fig. 3D-F). The black arrows in Fig. 3D-F indicated the area where particles were placed in the valley. As presented in Table III, the valley diameter, valley area and valley ratio were decreased following suction, regardless of the type of capsule. Furthermore, valley diameter, valley area and valley ratio of HPMC-Cap were significantly difference before TIO loading 
Table III. Valley diameter, valley area and valley ratio of each capsule type determined by atomic force microscopy images analysis.

\begin{tabular}{|c|c|c|c|c|c|c|}
\hline & \multicolumn{2}{|c|}{ Valley diameter, $\mu \mathrm{m}$} & \multicolumn{2}{|c|}{ Valley area, $\mu \mathrm{m}^{2}$} & \multicolumn{2}{|c|}{ Valley ratio, $\%$} \\
\hline & Before & After & Before & After & Before & After \\
\hline G-Cap & $0.59 \pm 0.26$ & $0.54 \pm 0.28$ & $0.28 \pm 0.22$ & $0.24 \pm 0.16$ & $4.60 \pm 0.75$ & $3.68 \pm 0.09$ \\
\hline PEG/G-Cap & $0.68 \pm 0.39$ & $0.64 \pm 0.36$ & $0.64 \pm 0.58$ & $0.46 \pm 0.32$ & $5.24 \pm 3.65$ & $4.51 \pm 0.54$ \\
\hline HPMC-Cap & $1.97 \pm 0.98$ & $1.06 \pm 0.40^{\mathrm{a}}$ & $2.05 \pm 1.89$ & $1.02 \pm 0.67^{\mathrm{a}}$ & $10.6 \pm 6.24$ & $6.39 \pm 0.16$ \\
\hline
\end{tabular}

Each value represents the mean \pm standard error of measurements. $n=120 .{ }^{a} \mathrm{P}<0.05$ vs. before TIO-loading. Before, before TIO-loading; After, after the suction TIO-loading capsule; G-Cap, gelatin capsule; PEG/G-Cap, gelatin capsule containing 5\% PEG4000 as plasticizer; HPMC-Cap, hydroxypropyl methylcellulose capsule; TIO, tiotropium bromide.
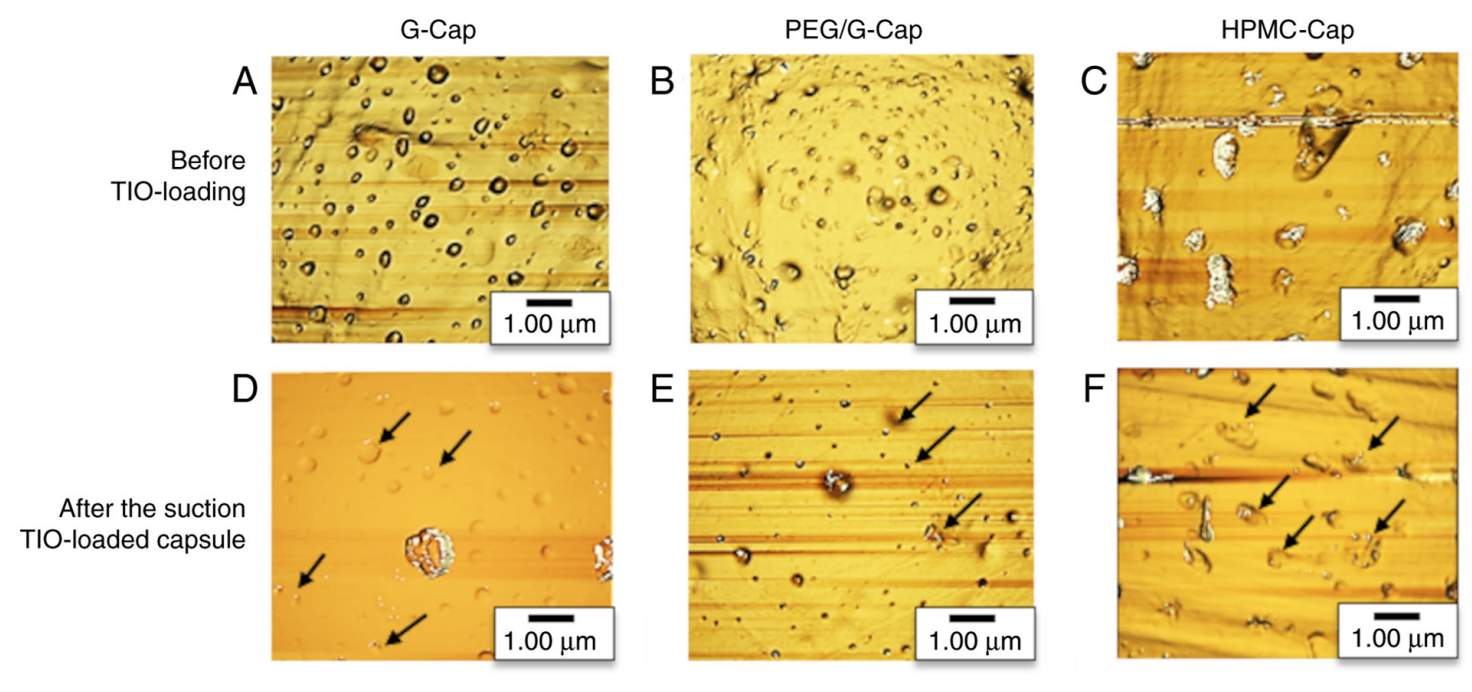

Figure 3. Comparison of the capsule inner surface structure by atomic force microscopy. (A-C) Inner surface of each capsule before drug loading. (D-F) Inner surface of each capsule after drug release. Black arrows in (D-F) indicate the area where particles are placed in the valley. G-Cap, gelatin capsule; PEG/G-Cap, gelatin capsule containing 5\% PEG4000 as plasticizer; HPMC-Cap, hydroxypropyl methylcellulose capsule; TIO, tiotropium bromide.

and after the suction of TIO-loaded capsules $(\mathrm{P}<0.05$, unpaired Student's t-test).

$3 D$ mapping analysis of the capsule inner surface structure and surface potential distribution. The 3D topography of the capsule inner surface and capsule inner surface potential distribution were evaluated using SPM-9700HT. The 3D topographies of the three capsule inner surfaces are presented in Fig. 4A-C. In Fig. 4A-C, the areas darker than background indicated the valleys, and the lighter areas indicated the mountains. These topographies revealed the distribution of valleys and mountains on the inner surface of each capsule. KFM images of the three capsule inner surfaces are shown in Fig. 4D-F. In these images, the red fraction indicates a higher potential and the blue fraction a lower potential. KFM images showed mixed high and low potential fractions in all three capsules. In addition, 3D topography and KFM image mapping were performed to confirm the relationship between the capsule inner surface structure and surface potential. The results of image mapping demonstrated that the distribution of valleys and mountains on the capsule inner surface corresponded to high and low potential fractions, respectively (Fig. 4G-I).
Comparison of inhalation performance. We evaluated drug release from capsule and lung drug delivery with different compositions using TSLI. Fig. 5A shows an image of the inner surface of each capsule before TIO loading and after the suction of TIO-loaded capsules. Images of TIO-loaded capsules after the suction demonstrated the adherence of TIO to the surface of capsule regardless of the capsule type. The results for inhalation performance parameters are presented in Fig. 5B and Table IV. The drug retention for capsule, RD, MB, ED, FPD and FPF were not significantly different between G-Cap, PEG/G-Cap and HPMC-Cap (NS, ANOVA).

\section{Discussion}

The advantages of capsule-based DPIs over other DPIs are as follows: Accurate and uniform drug delivery, ease to use for patients and the visual confirmation of whether a dose has been administered $(13,14)$. The inhalation performance of capsule-based DPIs is related to numerous factors, such as the inhalation patterns of patients (4-7), the inhalation devices (5) and the inhalation formulations (8). The inhalation performance of capsule-based DPIs was recently reported 

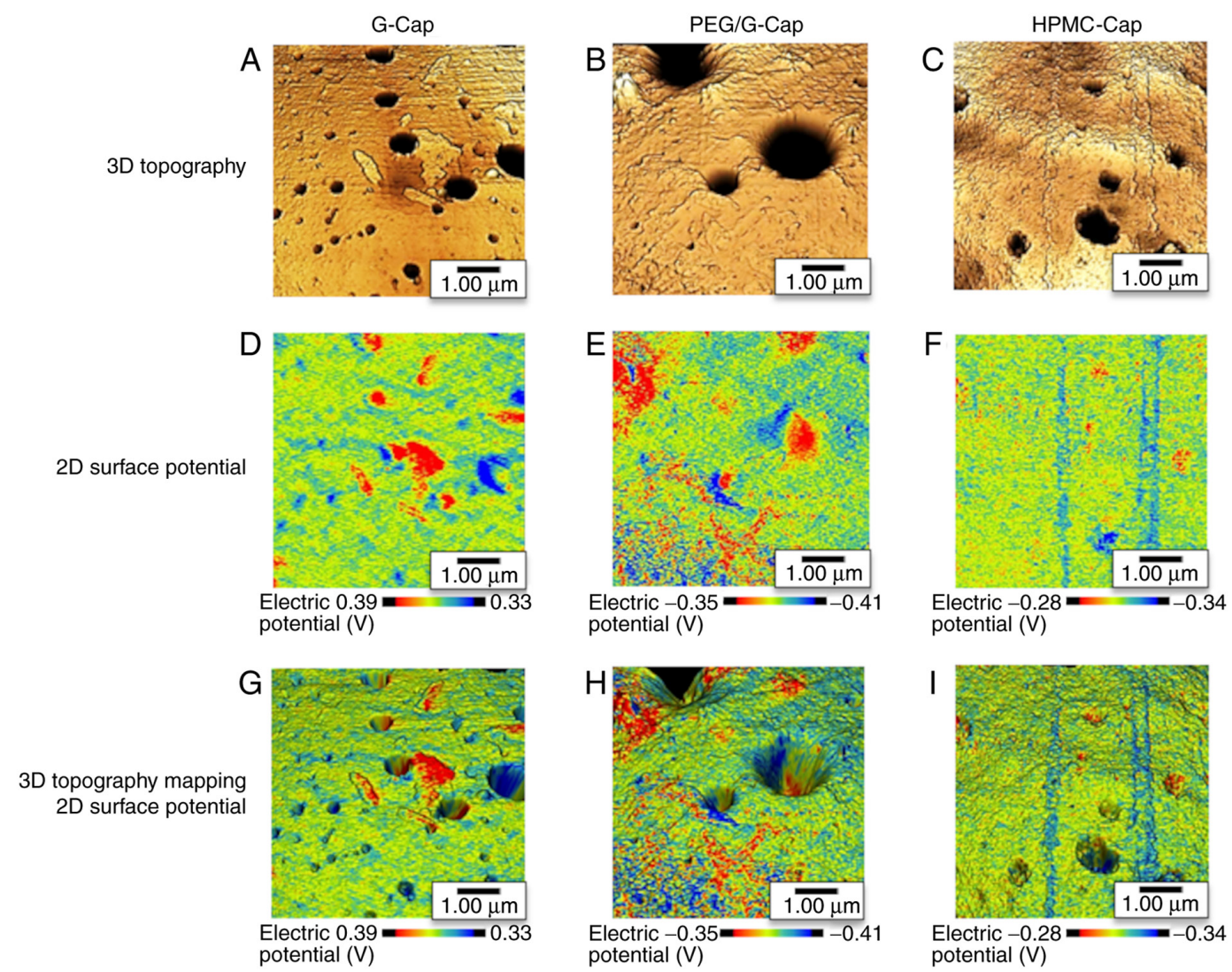

Figure 4. Atomic force microscopy, kelvin probe force microscopy and 3D mapping images of three capsule inner surface. (A-C) 3D topography of each capsule. (D-F) 2D surface potential of each capsule. (G-I) 3D topography mapping 2D surface potential of each capsule. G-Cap, gelatin capsule; PEG/G-Cap, gelatin capsule containing 5\% PEG4000 as plasticizer; HPMC-Cap, hydroxypropyl methylcellulose capsule; 2D, two dimensional; 3D, three dimensional.

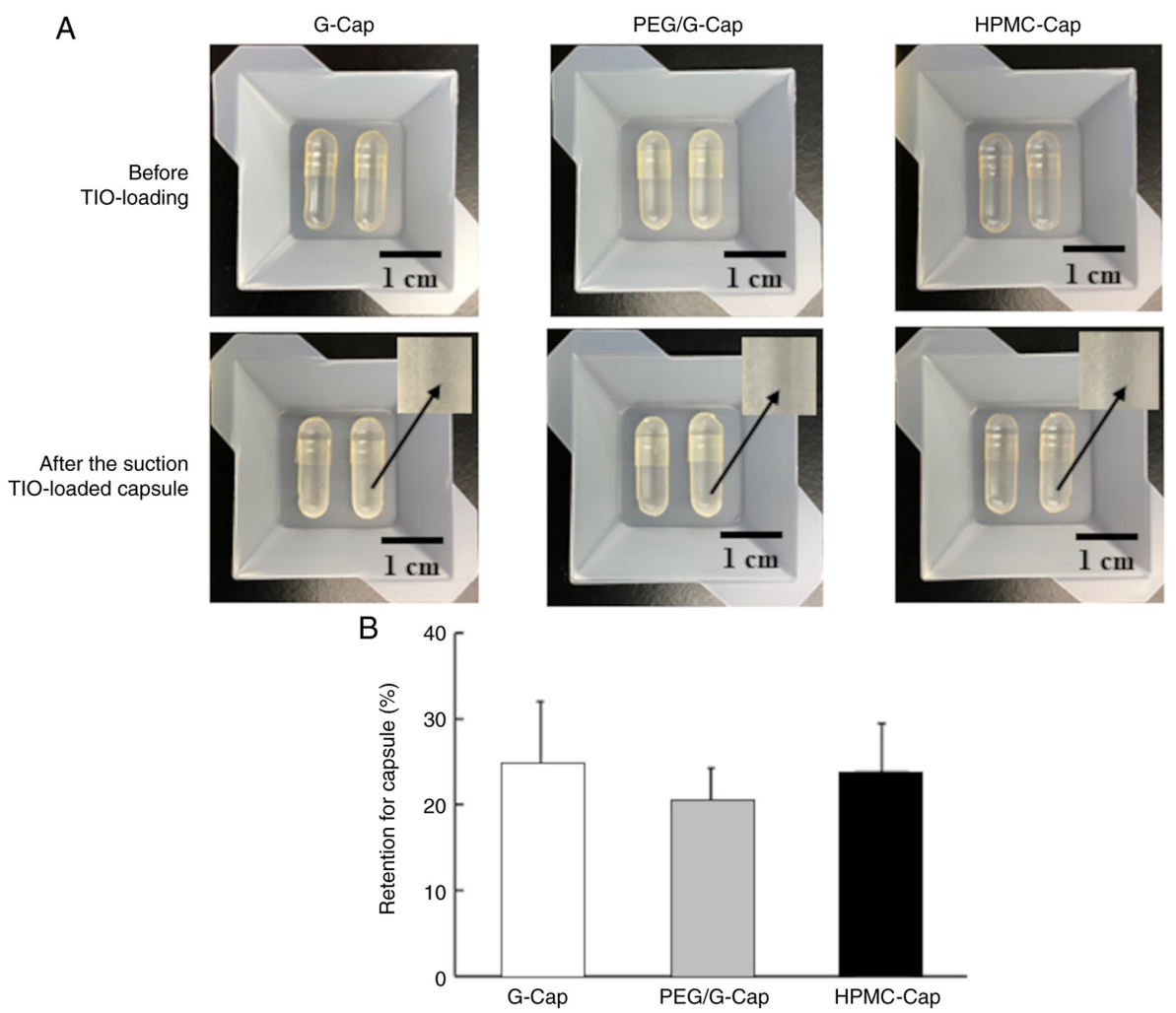

Figure 5. Evaluation of the drug retention for capsule using twin-stage liquid impinger. Inhaler used was Handihaler ${ }^{\circledR}$ (flow rate, 30 1/min). (A) Images of the capsule inner surface after drug release and (B) drug retention for capsule. Each value represents the mean \pm standard errors. Each experiment was repeated three times. G-Cap, gelatin capsule; PEG/G-Cap, gelatin capsule containing 5\% PEG4000 as plasticizer; HPMC-Cap, hydroxypropyl methylcellulose capsule; TIO, tiotropium bromide. 
Table IV. Inhalation performance of tiotropium bromide loaded in three capsules with different compositions using twin-stage liquid impinger.

\begin{tabular}{lccc}
\hline & G-Cap & PEG/G-Cap & HPMC-Cap \\
\hline Recovery dose, $\mu \mathrm{g}$ & $17.4 \pm 0.18$ & $16.8 \pm 0.42$ & $17.5 \pm 0.6$ \\
Mass balance, $\%$ & $96.9 \pm 0.98$ & $93.2 \pm 2.36$ & $97.5 \pm 3.54$ \\
Emitted dose, $\mu \mathrm{g}$ & $4.37 \pm 0.53$ & $4.67 \pm 0.14$ & $4.52 \pm 0.27$ \\
Fine particle dose, $\mu \mathrm{g}$ & $7.94 \pm 0.85$ & $7.54 \pm 0.45$ & $8.27 \pm 0.74$ \\
Fine particle fraction, $\%$ & $25.7 \pm 4.85$ & $28.3 \pm 4.50$ & $26.7 \pm 1.25$ \\
\hline
\end{tabular}

Each value represents the mean \pm standard error of measurements. $n=3$. G-Cap, gelatin capsule; PEG/G-Cap, gelatin capsule containing 5\% PEG4000 as plasticizer; HPMC-Cap, hydroxypropyl methylcellulose capsule.

to be affected by the capsule properties. In a previous study, inhalation performance was evaluated by filling five capsules with binary or ternary particles of different compositions and manufacturing methods, and the findings demonstrated that inhalation performance was affected by capsule compositions and manufacturing methods (22). However, few studies have investigated the effects of the physical properties of the capsule inner surface on inhalation performance. The present study aimed therefore to determine the capsule inner surface structures and surface potential distributions using the 3D mapping technologies, AFM topography and KFM imaging. Because the previous studies of capsule-based DPIs mainly focused on low resistance devices, not high resistance devices $(22,23)$, the present study investigated the effects of capsule physical properties on inhalation performance using a high resistance device of capsule-based DPIs.

To characterize the properties of the capsules, each capsule was evaluated by image analysis using ImageJ software. No significant difference was observed in the length diameter, width diameter, surface area and volume of each capsule, while the LOD of HPLC-Cap (5.0\%) was much lower than of G-Cap (14.3\%) and PEG/G-Cap (13.2\%; Table II). As the result of pierced capsule hole characteristics, there were no cracked holes in G-Cap, PEG/G-Cap and HPMC-Cap (Fig. 2). The capsule hole diameter and hole area of G-Cap tended to be larger than that of PEG/G-Cap and HPMC-Cap (Table II). The results of a previous study reported that different capsule compositions exhibit different levels of water content. The gelatin capsule and HPMC capsule demonstrated 13-16\% and $3-7 \%$ water content, respectively (27). Under low RH conditions, a decreased water content increases brittleness, with gelatin capsules being more sensitive to this effect (27). The capsule shell may therefore crack or fracture when the capsule is pierced, thereby affecting inhalation performance (28). In the present study, the capsule compositions were different, and it was expected that the capsules would crack during capsule piercing due to the difference in LOD. Although the hole diameter and hole area in each capsule was slightly different, no cracks occurred during capsule piercing. We considered that the capsule storage condition $\left(15-25^{\circ} \mathrm{C}, 40-50 \% \mathrm{RH}\right)$ and the piercing condition using two needles in the Handihaler ${ }^{\circledR}$ had a role in the lack of cracking.

To confirm the capsule inner surface structure, each capsule was observed using SPM. The inner surface structure on the center, top, bottom, left and right parts were observed in a previous experiment, and the results confirmed that there was no significant difference among these regions. The images from Fig. 3 were obtained by SPM of the central part of the capsule body. Numerous valleys and mountains were observed on each capsule inner surface before TIO loading, irrespective of the capsule composition (Fig. 3A-C). Following the suction TIO-loaded capsules, the inner surface of the capsule became smooth and TIO was placed in the valleys (Fig. 3D-F). To confirm whether the smoothness of the capsule inner surface was due to the TIO in the valleys, the valley diameter, valley area and valley ratio were compared before TIO loading and after the suction of TIO-loaded capsules (Table III). The valley diameter, valley area and valley ratio were slightly decreased after suction, regardless of the type of capsule. In particular, the valley diameter, area and ratio of the HPMC-Cap following the suction of TIO-loading were significantly different compared with these characteristics prior to TIO loading. The results suggested that, although the capsule had valley and mountain structure on the inner surface regardless of the capsule composition, the characteristics of valleys were affected by the capsule composition. Furthermore, drug particles may be trapped in the valleys on the capsule inner surface, which would affect the inhalation performance.

Subsequently, to confirm the electric properties of capsule inner surface, we evaluated the surface potential distribution using the KFM mode of SPM. KFM images demonstrated that regions with high and low potential fractions were mixed in each capsule (Fig. 4D-F). The 3D topography and KFM images mapping of capsules showed that the distribution valleys and mountains on the capsule inner surface corresponded to high and low potential fractions, respectively (Fig. 4G-I). Therefore, a local potential difference may have occurred due to the distribution of valleys and mountains on the capsule inner surface. These results suggested that the effect of the inner surface structure and the surface potential difference between the drug particles and the valleys may both contribute to the increase in TIO amount in the valleys.

To clarify the effects of the physical properties of the capsule inner surface on inhalation performance, the inhalation performance of each capsule type were evaluated using TSLI. The results of drug retention for capsule and lung drug delivery were not significantly different between 
G-Cap, PEG/G-Cap and HPMC-Cap (Fig. 5B and Table IV). These findings may be due to the fact that no significant difference in hole diameter and hole area between capsules was observed. In addition, drug particles were present in the inner surface valleys of each capsule, although their impact on drug retention for capsule and lung drug delivery was expected to be minimal. In the present study, we evaluated the inhalation performance using the high resistance device at an appropriate flow rate of $30 \mathrm{l} / \mathrm{min}$. The influence of inhalation device and flow rate on the drug release from capsule was therefore considered to be greater than that of the capsule compositions and physical properties of the capsule inner surface. In other words, at an appropriate flow rate using high resistance device, the physical properties of the capsule inner surface due to the capsule composition did not significantly affect the drug release from capsule and lung drug delivery. In this study, we particularly focused on the drug release from capsule, and inhalation performance was evaluated using TSLI. The TSLI is a simple inhalation characteristic apparatus that contains Throat, Stage 1 and Stage 2. The TSLI was set up so that drug particles reaching Stage 2 at a flow rate of $60 \mathrm{l} / \mathrm{min}$ showed a cut-off of less than $6.4 \mu \mathrm{m}$. Unfortunately, in the present study, the TSLI was examined at a flow rate of $30 \mathrm{l} / \mathrm{min}$, and the exact cut-off diameter could not be measured. Therefore, the stage grouping in the inhalation performance evaluation could not be performed, and the mass median aerodynamic diameter $(\mathrm{MMAD}) \pm$ geometric standard deviation (GSD) could not be calculated. To understand the exact aerodynamic differences in inhalation characterization, representative stage grouping data and MMAD $\pm \mathrm{GSD}$ are therefore needed $(29,30)$. Future investigation will evaluate the inhalation performance by calculating MMAD $\pm \mathrm{GSD}$ using Andersen cascade impactor, Next generation impactor and Multistage liquid impinger.

In summary, the present study investigated the effects of the physical properties of the capsule inner surface on drug release form capsules and lung drug delivery using AFM and a high resistance device. The results demonstrated that the capsule inner surface had many valleys and mountains regardless of the type of capsule. In addition, the valley and mountain areas on the capsule inner surface presented with potential fluctuations where there were significant differences in the surface structure. Following inhalation of capsule-based DPIs, the drug remained in valleys on the capsule inner surface; however, no significant difference was observed in drug retention for capsule and lung drug delivery. Therefore, drug release from capsule and lung drug delivery in capsule-based DPIs when a high resistance device, such as Handihaler ${ }^{\circledR}$, is used at an appropriately flow rate may have not significantly been affected by the physical properties of the capsule inner surface of the capsule due to capsule composition. However, since the present study only evaluated one DPI formulation and inhalation device, the effect of the physical properties of capsule inner surface on drug release and lung drug delivery remain currently unclear. Further studies using various DPI formulations, including indacaterol, glycopyrronium and andrographolide, and inhalation devices, such as Breezhaler ${ }^{\circledR}$, are needed to elucidate the relationship between the physical properties of capsule inner surface and inhalation performance.

\section{Acknowledgements}

We would like to thank Qualicaps, Co., Ltd., for supplying G-Cap, PEG/G-Cap and HPMC-Cap, and Shimadzu Corp. for providing AFM topography and KFM images of each capsule surface using SPM-9700HT. This study was partly supported by the Hosokawa Powder Technology Foundation.

\section{Funding}

The present study was supported partly by The Mochida Memorial Foundation for Medical and Pharmaceutical Research (grant no. 14).

\section{Availability of data and materials}

All data generated or analyzed during this study are included in this published article.

\section{Author's contributions}

$\mathrm{HO}$ and MM performed the experiments and were the major contributors to writing the manuscript. MY, SA and KI analyzed and interpreted the data. HO and NN made substantial contributions to the conception and design of the study. $\mathrm{NN}$ designed the experiments and gave final approval for the version of the manuscript to be published. MM and MY confirm the authenticity of all the raw data. All authors read and approved the final manuscript.

\section{Ethic approval and consent to participate}

Not applicable.

\section{Patient consent for publication}

Not applicable.

\section{Competing interests}

The authors declare that they have no competing interests.

\section{References}

1. Claus S, Weiler C, Schiewe J and Friess W: How can we bring high drug doses to the lung? J Pharm Biopharm 86: 1-6, 2014.

2. Yang MY, Chan JG and Chan HK: Pulmonary drug delivery by powder aerosols. J Control Rel 193: 228-240, 2014.

3. Tonnis WF, Lexmond AJ, Frijlink HW, de Bore AH and Hinrichs WL: Devices and formulations for pulmonary vaccination. Expert Opin Drug Deliv 10: 1383-1397, 2013.

4. Bouwmeester C, Kraft J and Bungay KM: Optimizing inhaler use by pharmacist-provided education to community-dwelling elderly. Respir Med 109: 1363-1368, 2015.

5. Broeders ME, Vincken W, Corbetta L and Group AW: The ADMIT series-Issues in inhalation therapy. 7. Ways to improve pharmacological management of COPD: The importance of inhaler choice and inhalation technique. Prime Care Respir J 20: 338-343, 2011.

6. Cochrane MG, Bala MV, Downs KE, Mauskopf J and Ben-Joseph RH: Inhaled corticosteroids for asthma therapy: Patient compliance, devices, and inhalation technique. Chest 117: 542-550, 2000.

7. Baily MM, Gorman EM, Munson EJ and Berkland C: Pure insulin nanoparticle agglomerates for pulmonary delivery. Lungmuir 24: 13614-13620, 2008. 
8. Weer JG and Miller DP: Formulation design of dry powders for inhalation. J Pharm Sci 104: 3259-3288, 2015.

9. Stocks J and Hislop AA: Structure and function of the respiratory system: Developmental aspects and their relevance to aerosol therapy. In: Drug Delivery to the Lung: Clinical Aspects. Bisgaard H, O'Callaghan C, Smaldone CG (eds.) Marcel Dekker, Inc., New York, NY, pp47-104, 2002.

10. Lavorini F, Pistolesi M and Usmani OS: Recent advances in capsule-based dry powder inhaler technology. Multidiscip Respir Med 12: 11, 2017.

11. Berkenfeld K, Lamprecht A and McConville JT: Devices for dry powder drug delivery to the lung. AAPS PharmSciTech 16: 479-490, 2015

12. Faulhammer E, Flink M, Liusa M, Lawrence SM, Biserni S, Calzolari V and Khinast JG: Low-dose capsule filling of inhalation products: Critical material attributes and process parameters. Int J Pharm 473: 617-626, 2014.

13. Wauthoz N, Hennia I, Dejaeger B, Ecenarro S and Amighi K Proposed algorithm for healthcare professionals based on product characteristics and in vitro performances in different use conditions using formoterol-based marketed products for inhalation. Int J Pharm 530: 415-429, 2017.

14. Smith IJ, Bell J, Bowman N, Everard M, Stein S and Weer JG: Inhaler devices: What remains to be done? J Aerol Med Pulm Drug Deliv (Suppl 2): S25-S37, 2010.

15. Steckel $\mathrm{H}$ and Muller BW: In-vitro evaluation of dry powder inhalers 1: Drug deposition of commonly used devices. Int J Phar 154: 19-29, 1997.

16. Vidgren M, Kärkkäinen A, Karjalainen $\mathrm{P}$, Paronen $\mathrm{P}$ and Nuutinen J: Effect of powder inhaler design on drug deposition in the respiratory tract. Int J Pharm 42: 211-216, 1988.

17. Chew NY, Shekunov BY, Tong HH, Chow AH, Savage C, $\mathrm{Wu} \mathrm{J}$ and Chan $\mathrm{H}$ : Effect of amino acids on the dispersion of disodium cromoglycate powders. J Pharm Sci 94: 2289-2300, 2005.

18. Otake H, Okuda T and Okamoto H: Development of spray-freeze dried powders for inhalation with high inhalation performance and antihygroscopic property. Chem Pharm Bull (Tokyo) 64 239-245, 2016

19. Li HY, Seville PC, Williamson IJ and Birchall JC: The use of amino acids to enhance the aerosolisation of spray-dried powders for pulmonary gene therapy. J Gene Med 7: 343-353, 2005.

20. Ungaro F, Giovino C, Coletta C, Sorrentino R, Miro A and Quaglia F: Engineering gas-foamed large porous particles for efficient local delivery of macromolecules to the lung. Eur J Pharm Sci 41: 60-70, 2010.
21. DuanJ,VogtFG,LiX,Hayes JrDand MansourHM:Design,characterization, andaerolization of organicsolutionadvanced spray-dried moxifloxacin and ofloxacin dipalmitoylphosphatidylcholine (DPPC) microparticulate/nanoparticulate powders for pulmonary inhalation aerosol delivery. Int J Nanomedicine 8: 3489-3505, 2013

22. Wauthoz N, Hennia I, Ecenarro S and Amighi K: Impact of capsule type on aerodynamic performance of inhalation products: A case study using a formoterol-lactose binary or ternary blend. Int J Pharm 553: 47-56, 2018.

23. Saleem IY, Diez F, Jones BE and Polo L: Investigation on the aerosol performance of dry powder inhalation hypromellose capsules with different lubricant levels. Int J Pharm 492: 258-263, 2015.

24. Horhota ST, van Noord JA, Verkleij CB, Bour LJ, Sharma A, Trunk $\mathrm{M}$ and Cornelissen PJ: In vitro, pharmacokinetic, pharmacodynamic, and safety comparisons of single and combined administration of Tiotropium and Salmeterol in COPD patients using different dry powder inhalers. AAPS J 17: 871-880, 2015.

25. Hira D, Koide H, Nakamura S, Okada T, Ishizeki K, Yamaguchi M, Koshiyama S, Oguma T, Ito K, Funayama S, et al: Assessment of inhalation flow patterns of soft mist inhaler co-prescribed with dry powder inhaler using inspiratory flow meter for multi inhalation devices. PLoS One 13: e0193082, 2018.

26. García R and Pérez R: Dynamic atomic force microscopy methods. Surf Sci Rep 47: 197-301, 2002

27. Nagata S: Gellulose capsules-an alternative to gelatin. In: Biolodical Polymers and Polymer Therapeutics. Chiellini E, Sunamoto J, Migliaresi C, Ottenbrite RM, Cohn D (eds). Kluwer Academics/Plenum Publishers, New York, NY, pp 53-62, 2001.

28. Coate MS, Fletcher DF, Chan HK and Raper JA: The role of capsule on the performance of a dry powder inhaler using computational and experimental analysis. Pharm Res 22: 923-932, 2005.

29. Mehta PP, Kadam SS and Pawar AP: Effect of USP induction ports and modified glass sampling apparatus on aerosolization performance of lactose carrier-based fluticasone propionate dry powder inhaler. J Drug Deliv Sci Technol 58: 101794, 2020.

30. Timsina MP, Martin GP, Marriott C, Ganderton D and Yianneskis M: Drug delivery to the respiratory tract using dry powder inhalers. Int J Pharm 101: 1-13, 1994.

(i) $\odot$ This work is licensed under a Creative Commons Attribution-NonCommercial-NoDerivatives 4.0 International (CC BY-NC-ND 4.0) License. 